\title{
Removal of Singularities from Taylor Series
}

K. La Mon

August 1989

Prepared for the U.S. Department of Energy under Contract Number DE-AC03-76SF00098. 
LBL-27689

ESG Note-77

Removal of Singularities From Taylor Series

\author{
Kenneth La Mon \\ Exploratory Studies Group \\ Accelerator and Fusion Research Division \\ Lawrence Berkeley Laboratory \\ 1 Cyclotron Road \\ Berkeley, CA 94720
}

August 1989

* This work was supported by the Director, Office of Energy Research, Office of High Energy and Nuclear Physics, High Energy Physics Division, of the U.S. Department of Energy under Contract No. DE-AC03-76SF00098. 


\title{
Removal of Singularities From Taylor Series
}

\author{
By Kenneth La Mon
}

\begin{abstract}
A mathematical procedure is described whereby the radius of convergence of a Taylor series can be increased through the inclusion of complex poles in a rational approximation. Computer results show that this technique is quite independent of the asymptotic limit of the power series and only depends on the positions of the singularities. Aside from the applications in one variable, this method vastly improves perturbative solutions to symplectic, dynamical mappings in many dimensions by removing resonances in the complex plane.
\end{abstract}

\section{Introduction}

Whenever a Taylor series is substituted for a nonentire analytic function, the radius of convergence of that series is determined by the distance from the origin of the expansion to the nearest singularity in the complex plane. For example, the function

$$
(1+z)^{1 / 2}
$$

is well behaved near $\mathrm{z}=1$ but, because its first derivative becomes infinite at the branch point $\mathrm{z}=-1$, its Taylor expansion about $z=0$ diverges for $|z|>1$. A better approximation near $z=1$ can be found by multiplying and dividing this series by $(1+z)$ thereby explicitly including the singularity at $z=-1$ in the new approximation.

$$
\begin{aligned}
1+\frac{1}{2} z-\frac{1}{8} z^{2}+\ldots & \rightarrow \frac{(1+z)\left(1+\frac{1}{2} z-\frac{1}{8} z^{2}+\ldots\right)}{(1+z)} \\
& =\frac{\left(1+\frac{3}{2} z+\frac{3}{8} z^{2}+\ldots\right)}{(1+z)}
\end{aligned}
$$

Now the Taylor series in the numerator is that of $(1+z)^{3 / 2}$, and here the singularity is in the second derivative rather than the first. This Taylor series still diverges for $|z|>1$ but, because the singularity appears at a higher order in the approximation, the divergence is not as strong. Continuing this process indefinitely yields a series for which the singularity is far enough away not to be felt. Of course, in practice, one only deals with finite series so that moving the singularity to higher 
and higher order means that we can keep more terms in the Taylor expansion in the numerator. That is:

$$
\sqrt{1+z}=\lim _{\mathrm{N} \rightarrow \infty} \frac{\left[\left(1+\frac{1}{2} z-\frac{1}{8} z^{2}+\ldots z^{N}\right)(1+z)^{N-1}\right]_{N}}{(1+z)^{N-1}}
$$

Here the subscript $\mathrm{N}$ in the numerator means truncate the resulting bracketed polynomial at order $\mathrm{N}$.

By no means does this heuristic argument constitute a proof, the real confirmation coming from extensive computer study over a wide range of sample functions. This work was facilitated by and, in part, motivated by the Differential Algebra (DA) package of $M$. Berz. ${ }^{1}$ Using this set of FORTRAN subroutines one can quickly generate the Taylor series of simple functions to arbitrary order in many dimensions and, in addition, manipulate these series at finite order through multiplication, division, etc..

Because the new rational approximation contains information about the function that the old series did not have, specifically the positions of poles, it actually converges faster inside the radius of convergence of the other series. Table 1 gives a comparison of relative errors [ relative error $=$ (approx.- exact value)/exact value ] for fifteenth order series for the function:

$$
\sqrt{1+z+z^{2}} \rightarrow \frac{\left[\left(1+\frac{1}{2} z+\frac{3}{8} z^{2}+\ldots z^{N}\right)\left(1+z+z^{2}\right)^{k}\right]_{N}}{\left(1+z+z^{2}\right)^{k}}
$$

In this case the function has poles at $z=\exp ( \pm 2 \pi i / 3)$. The rule for removing multiple poles is to choose $k$ to be the greatest integer less than $\mathrm{N}$ divided by the number of poles.

Table 1: Relative Errors $\left(1+z+z^{2}\right)^{1 / 2}$

$\begin{array}{cc}\text { zaylor Series } & \text { Rational Approx. } \\ \mathrm{N}=15 & \mathrm{~N}=15, \mathrm{k}=7\end{array}$

\begin{tabular}{rcc}
\hline & & \\
0.2 & $2.48(-14)$ & $2.33(-14)$ \\
0.4 & $1.67(-9)$ & $2.49(-10)$ \\
0.6 & $1.02(-6)$ & $2.79(-8)$ \\
0.8 & $9.18(-5)$ & $4.58(-7)$ \\
1.0 & $2.88(-3)$ & $3.40(-6)$ \\
1.5 & $*$ & $6.03(-5)$ \\
2.0 & $*$ & $2.85(-4)$ \\
3.0 & $*$ & $1.42(-3)$ \\
10.0 & $*$ & $1.36(-2)$ \\
\hline
\end{tabular}

Table 2 shows the diminishment of relative error for this same function as $\mathrm{N}$, the order of the expansion, is increased. 
Table 2: Relative Errors $\left(1+z+z^{2}\right)^{1 / 2}, z=1.5$

N, k Rational Approx.

$\begin{array}{rl}5,2 & 1.64(-2) \\ 10,4 & 1.37(-3) \\ 15,7 & 6.03(-5) \\ 20,9 & 5.44(-6) \\ 30,14 & 2.50(-8) \\ 40,19 & 1.22(-10)\end{array}$

Application to Dynamical Systems

Using a Poincaré surface of section one can study the dynamics of trajectories in the neighborhood of a closed orbit $\Gamma$ in terms of a discrete transfer mapping $\mathrm{Mz}_{i}=z_{i+1}$ where $M$ gives the position of the $(i+1)^{\text {th }}$ piercing of the S.O.S. in terms of the position of the $i^{\text {th }}$ piercing. The closed orbit $\Gamma$ is just a fixed point of $M$ taken to be at the origin of the reduced phase space. For stable Hamiltonian systems, the linear mapping will simply be a rotation about the origin. As nonlinearities are added, features such as chaos and island chains immediately arise.

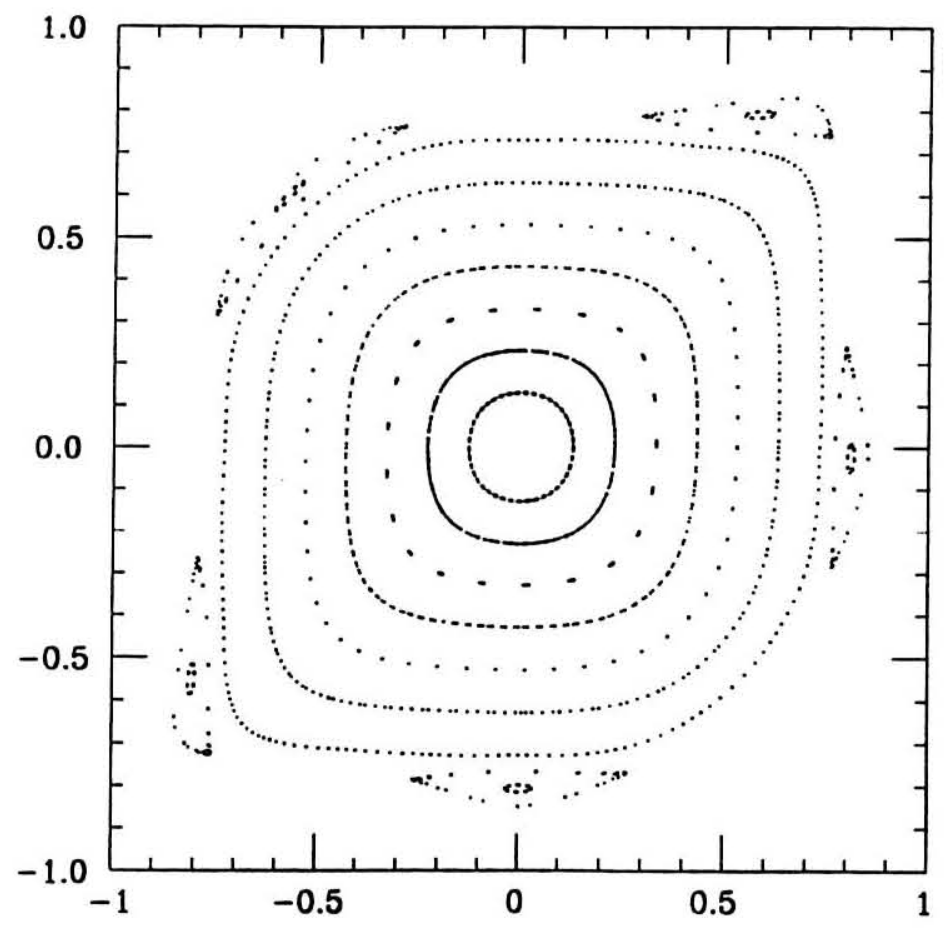

Figure 1: A plot of the iterates of Eq. (1) with $\mu=0.245(2 \pi)$ 
Figure 1 shows the iterates, for various initial conditions, of the nonlinear Hamiltonian mapping:

$$
\begin{aligned}
& \mathrm{X}=\mathrm{x} \cos \mu+p \sin \mu \\
& \mathrm{P}=-\mathrm{x} \sin \mu+\mathrm{p} \cos \mu+\mathrm{x}^{3}
\end{aligned}
$$

In this case the constant tune $\mu$ was chosen to be $(0.245)(2 \pi)$ in order to excite the $\mathrm{m}=1 / 4$ resonance to which this mapping is highly sensitive.

One method for constructing a perturbative solution to such a Hamiltonian mapping involves factoring it using a similarity transformation.

$$
\begin{aligned}
& M=A^{-1} N A \\
& N=N(J) \quad J=\left(x^{2}+p^{2}\right) / 2
\end{aligned}
$$

Here the map $\mathrm{N}$ is chosen to depend only on the radial variable $\mathrm{J}$. Hence the transformation $A$ sends us to a set of coordinates in which the motion in the phase plane is simply a rotation at a constant amplitude-dependent frequency. To find out where an initial vector $\left(x_{0}, p_{0}\right)$ will be after $n$ iterations of the map $M$ we simply apply the transformation $A$, rotate by a constant angle, and then transform back using $A^{-1}$.

The shapes of the invariant curves of $M$ are given by the function $\mathrm{A}^{-1} \mathrm{~J}=$ constant since

$$
\begin{aligned}
\mathrm{MA}^{-1} \mathrm{~J} & =\mathrm{A}^{-1} \mathrm{NJ} \\
& =\mathrm{A}^{-1} \mathrm{~J} .
\end{aligned}
$$

The method for constructing this factorization involves Lie transforms and is quite beyond the scope of this paper although several good sources exist that describe it $2,3,4$. Strictly speaking, the asymptotic series generated by this factorization cannot converge. Instead, for near-integrable systems, the approximation keeps getting better up to a certain order in the expansion after which additional terms make the series diverge.

Characteristically, however, the divergences that first arise do not seem to be due to any fundamental property of the motion such as chaos. Rather the power series solution generated resembles the Taylor series of a function with complex poles. Using the method described previously for obtaining a rational approximation from a Taylor series we can fix up these divergent solutions.

Finding the positions of the singularities in the case of dynamical mappings is actually quite straightforward since the mapping $\mathrm{N}$ gives the frequency of the motion as a function of amplitude in the transformed space. When this frequency is a 
rational multiple of $2 \pi$ we are at resonance, and for small low-order fractions, the resonance will cause our series to be singular.

\section{Results}

If we look at the effect of applying the transformation $A^{-1}$ to the particles tracked through $M$, we see that the invariant tori in the original space are transformed into circles in the new space. This provides a visual way of testing the perturbation series using a computer.

Figure $2 a$ shows the orbits of Figure 1 once they have been mapped through the 15 th order polynomial $A^{-1}$. Notice that the tori are transformed into circles up to a radius of about $r=0.2$; after that the transformation fails horribly. Since there is no discontinuity in the original space, like an island chain, this singularity is presumably removable.

From the mapping $\mathrm{N}$ we get a formula for the tune shift with amplitude in the new space:

$$
\omega=0.245(2 \pi)-(3 / 4) \mathrm{J}+O(4)
$$

The resonance occurs when $\omega=0.25(2 \pi)$ or

$$
(2 \pi / 75)+x^{2}+p^{2}=0
$$

To patch up the 15th order solution multiply and divide by $k=7$ powers of eq. (2) and truncate at order 15. Figure $2 \mathrm{~b}$ shows the $\mathrm{N}=19, k=9$ rational function solution which is completely purged of this singularity.
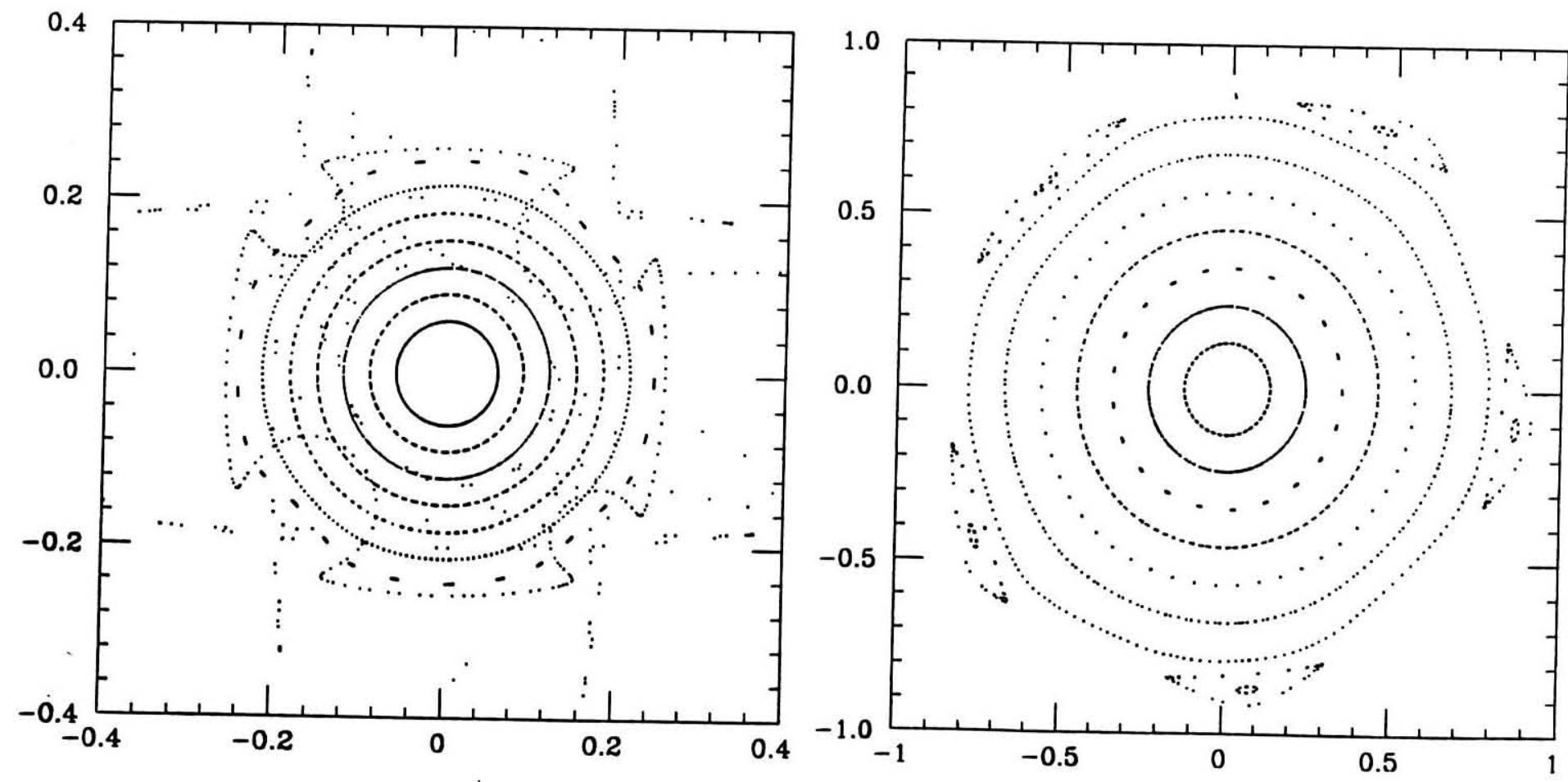

Figure 2: Trajectories of Eq. (1) with $\mu=0.245(2 \pi)$ after 
being acted on by a) 15 th order polynomial $A^{-1}$ b) $N=19, k=9$ rational function $\mathrm{A}^{-1}$.

In an earlier paper the author showed ${ }^{5}$ that by altering the formulation of the similarity transformation slightly one may construct a solution that converges best at a specified radial distance from the origin. Figure $3 a$ shows the iterates of the map (1) but, in this case, the constant tune $\mu$ was chosen to be $0.255(2 \pi)$. Figure $3 b$ shows the results from applying to these points the 10 th order polynomial map $A^{-1}$, derived about the radius $r=0.75$, outside the large, four-turn island chain. As the order of the transformation is increased, the small "spurs" on the edge of the picture grow until by 15 th order, in Figure $4 a$, they dominate.

Figure $4 \mathrm{~b}$ shows what happens when this $\mathrm{N}=15$ polynomial solution is converted to a $\mathrm{N}=15, \mathrm{k}=7$ rational approximation using:

$$
0.25(2 \pi)=0.222(2 \pi)+0.033(2 \pi) \alpha^{2}-(3 / 4) J+0(4) .
$$

Figure $4 \mathrm{~b}$ is actually a composite picture; the three outermost orbits of Figure $4 \mathrm{a}$ were remapped through the rational transformation whereas the nondivergent trajectories were left alone. In order to create the original radially displaced factorization it was necessary to introduce the ordering parameter $\alpha$ above, set equal to 1 at the end of the calculation. This parameter is partly responsible for creating the singularity and must be included for its removal.
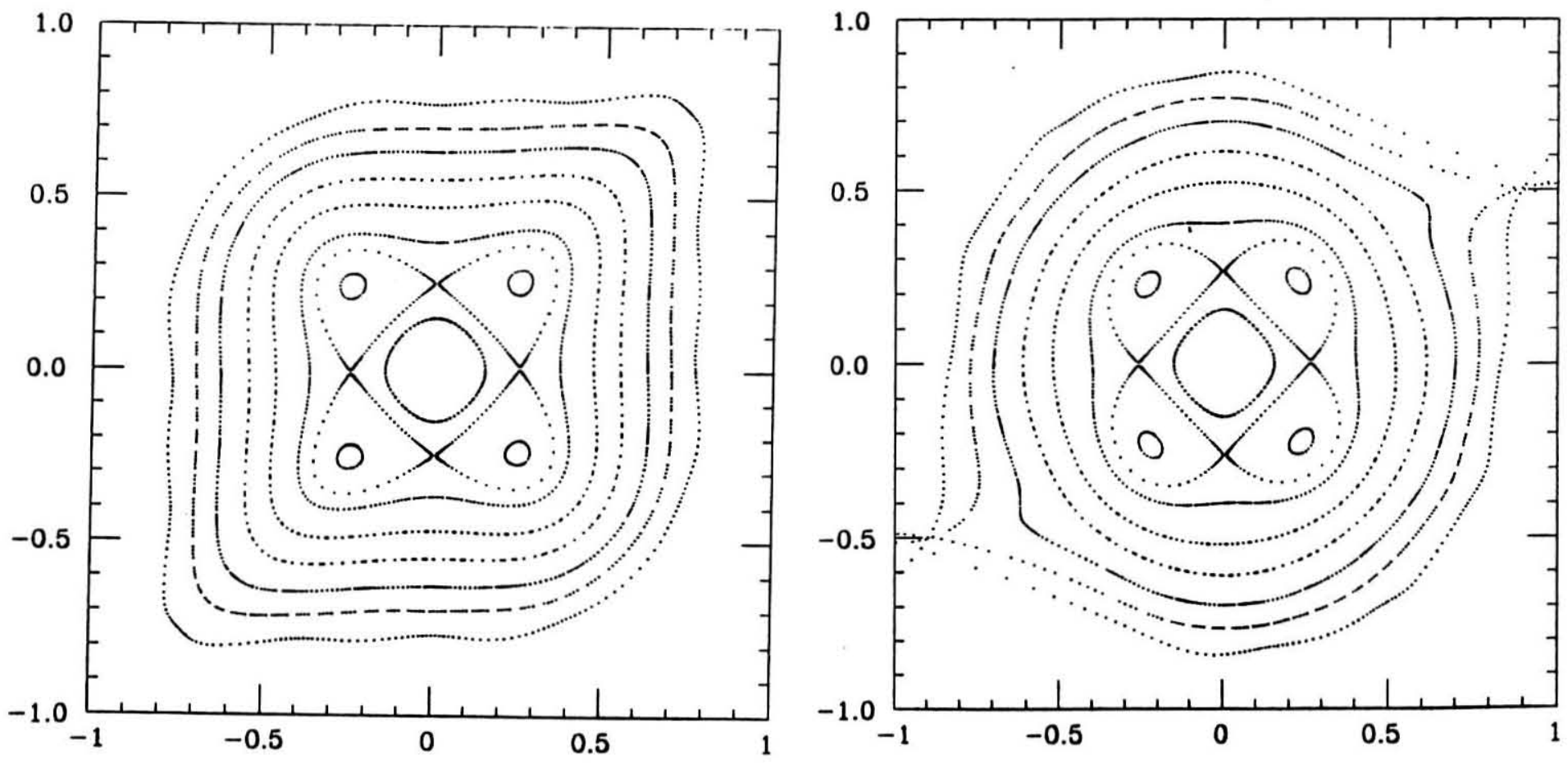

Figure 3: a) A plot of the iterates of Eq. (1) with $\mu=0.255(2 \pi)$ b) These same trajectories after being acted on by a loth order polynomial $A^{-1}$ 

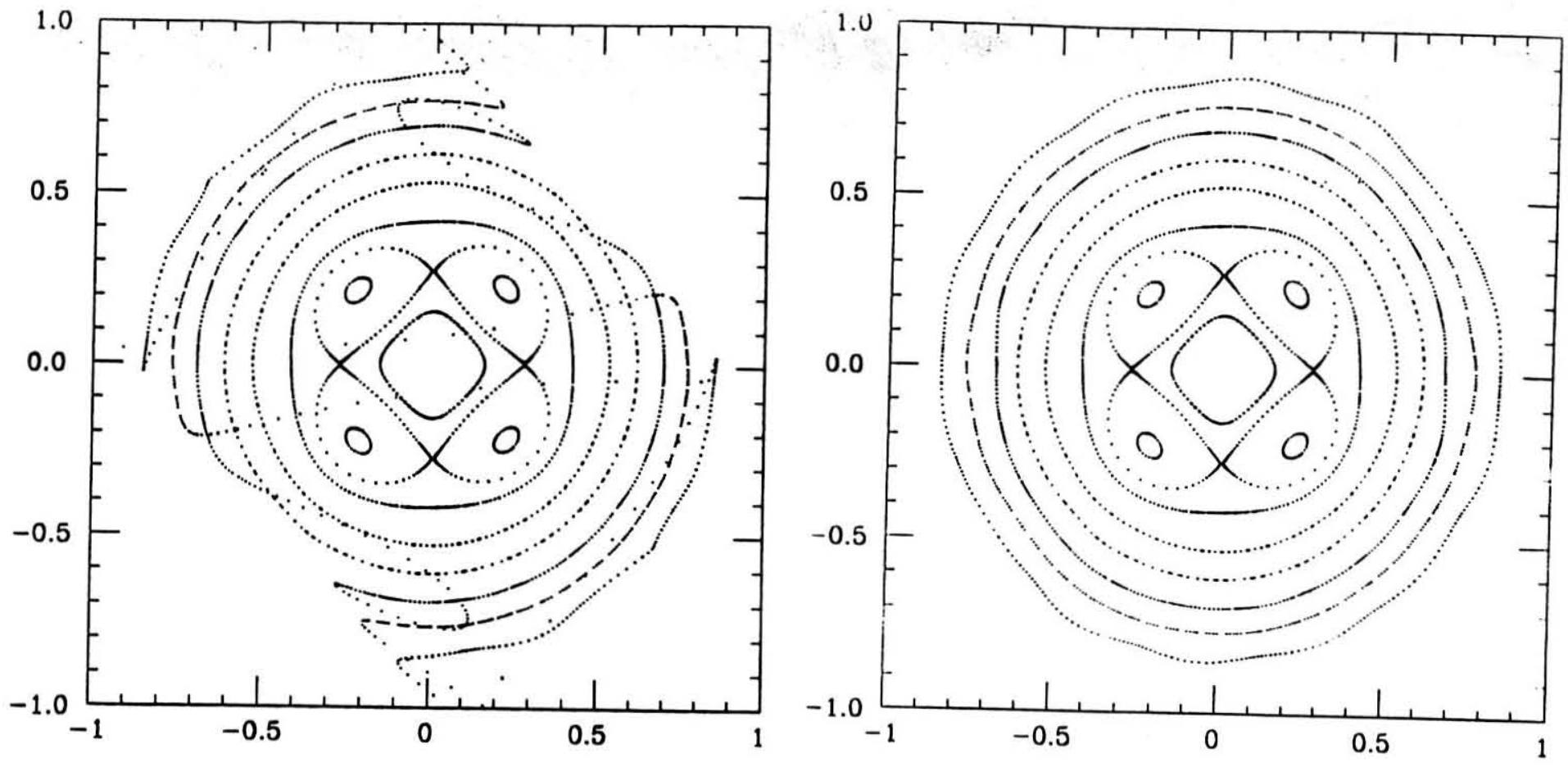

Figure 4: a) Trajectories of Fig. 3a after being mapped through a 15 th order polynomial $A^{-1}$. b) Same as $4 \mathrm{a}$ but with the three outer trajectories mapped through an $\mathrm{N}=15, \mathrm{k}=7$ rational approximation.

The removal of resonances in higher dimensions proceeds in exactly the same manner as before. In four variables, to remove a resonance of the form:

$$
1 \omega_{x}+m \omega_{y}=n
$$

multiply and divide by $k$ factors of

$$
1 \frac{\partial \mathrm{H}}{\partial J_{x}}+m \frac{\partial H}{\partial J_{y}}+n=0
$$

where $\mathrm{H}$ is a polynomial, derived from $\mathrm{N}$, describing tune shift with amplitude. 


\section{References}

[1] M. Berz, Particle Accelerators 24, 109 (1989).

[2] A.J. Dragt and J.M. Finn, J. Math. Phys. 172215 (1976).

[3] A.J. Dragt and J.M. Finn, J. Math. Phys. 202649 (1979).

[4] E. Forest, M. Berz and John Irwin, Particle Accelerators 24, 91 (1989).

[5] K. La Mon, Submitted Journal of Physics A (1989). 